\title{
ON THE REFLECTION PRINCIPLE IN SEVERAL COMPLEX VARIABLES
}

\author{
S. M. WEBSTER
}

\begin{abstract}
The edge-of-the-wedge theorem is used to extend a biholomorphic map across a nondegenerate real analytic boundary in $\mathbf{C}^{n}$ under some differentiability assumption at the boundary.
\end{abstract}

The classical theorem of $H$. A. Schwarz says that if a function is holomorphic in a plane region and extends continuously to an analytic arc in the boundary of the region, then it extends holomorphically across the arc. An analogue in several complex variables is C. Fefferman's theorem [1] that a biholomorphic map between two bounded strongly pseudoconvex domains with real analytic boundaries extends biholomorphically across the boundary. Recently, H. Lewy [2] has given an elementary proof of a local variant of this result for a holomorphic map which is continuously differentiable to the boundary. See also Pinčuk [4].

The purpose of this note is to give a similar elementary proof of extension of a biholomorphic map, assuming some regularity to the boundary. The main idea is to use the edge-of-the-wedge theorem. The distributional version of this theorem permits the reduction of the differentiability assumptions somewhat. We also introduct a natural reflection associated to a nondegenerate analytic real hypersurface.

Let $M$ and $M^{\prime}$ be analytic real hypersurfaces having nondegenerate Levi forms and bounding on domains $D$ and $D^{\prime}$, respectively, in $\mathbf{C}^{n}, n>2$. Let $f$ : $D \rightarrow D^{\prime}$ be a biholomorphic map which extends continuously to $M$ and maps $M$ into $M^{\prime}$.

We first define a reflection mapping, i.e. a local anti-holomorphic involution. Let $\mathbf{C}^{n} \times \mathbf{P}_{n-1}$ be the $(2 n-1)$-dimensional complex manifold of tangent $(n-1)$-planes in $\mathbf{C}^{n}$. We take as local coordinates $z_{\alpha}, z_{n}, p_{\alpha}, 1 \leqslant \alpha \leqslant$ $n-1 . M$ is given locally by a real analytic equation

$$
r(z, \bar{z})=0, \quad d r \neq 0 .
$$

Varying $z$ and $\bar{z}$ independently, we define local complex hypersurfaces $Q_{w}=\{z: r(z, \bar{w})=0\}$ for points $w$ near $M$. The reality condition on $r$ implies that $z$ is in $Q_{w}$ if and only if $w$ is in $Q_{z}$. Let $T_{z} Q_{w}$ denote the tangent plane to $Q_{w}$ at $z$. It exists for $z$ and $w$ near a point of $M$, since $\partial r(z, \bar{w}) \neq 0$. For

Received by the editors February 10, 1978.

AMS (MOS) subject classifications (1970). Primary 32H99.

Key words and phrases. Nondegenerate Levi form, biholomorphic map. 
$r(z, \bar{w})=0$ define

$$
\tau\left(z, T_{z} Q_{w}\right)=\left(w, T_{w} Q_{z}\right)
$$

Alternately, $\tau(z, p)=(w, q)$ is defined implicitly by the equations

$$
\begin{aligned}
& r(z, \bar{w})=0, \\
& r_{\alpha}(z, \bar{w})+p_{\alpha} r_{n}(z, \bar{w})=0, \\
& r_{\bar{\alpha}}(z, \bar{w})+\bar{q}_{\alpha} r_{\bar{n}}(z, \bar{w})=0,
\end{aligned}
$$

where $1 \leqslant \alpha \leqslant n-1$, the subscripts on $r$ denote partial derivatives with respect to the $z$ and $\bar{w}$ variables, and we assume $r_{n} \neq 0$. The Jacobian determinant of this system with respect to the variables $\bar{w}, \bar{q}$ is

$$
\left(r_{\bar{n}}\right)^{n-1} \operatorname{det}\left[\begin{array}{cc}
r_{\bar{\beta}} & r_{\bar{n}} \\
r_{\alpha \bar{\beta}}+p_{\alpha} r_{n \bar{\beta}}, & r_{\alpha \bar{n}}+p_{\alpha} r_{n \bar{n}}
\end{array}\right] .
$$

With $z=w \in M$ and taking into account (1), we see that this is a nonzero multiple of the Levi determinant. By our assumption on the Levi form it follows that $\tau$ is defined in a neighborhood of $(z, p), z \in M, p=H_{z} M$, by the implicit function theorem. Also from the form of the equations (1) it follows that $\tau$ is anti-holomorphic, $\tau^{2}=1$, and that the fixed point set of $\tau$ is precisely $\tilde{M}$, the set of holomorphic tangent planes $H_{z} M$ to $M$. This gives the following lemma.

LEMMA. The real $(2 n-1)$-dimensional manifold $\tilde{M}$ of complex tangent planes to $M$ is totally real in the space $\mathbf{C}^{n} \times \mathbf{P}_{n-1}$ of all tangent $(n-1)$-planes.

Furthermore, one can readily verify that if $M$ is twice continuously differentiable then $\tilde{M}$ is totally real if and only if $M$ has nondegenerate Levi form.

It follows that any point $(z, p)$ in $\tilde{M}$ has a holomorphic coordinate system in which $\tilde{M}$ corresponds to $\mathbf{R}^{2 n-1} \subset \mathbf{C}^{2 n-1}$. For an explicit construction we assume that $z=p=0$ and that $M$ is given locally by

$$
r=\sum \varepsilon_{\alpha}\left|z_{\alpha}\right|^{2}+F\left(z_{\alpha}, \bar{z}_{\alpha}, x_{n}\right)-y_{n}=0,
$$

where $\varepsilon_{\alpha}= \pm 1, F$ is real analytic and vanishes to order three at the origin, and $D$ is given by $r<0 . M$ is parameterized analytically by $z_{\alpha}=x_{\alpha}+i y_{\alpha}$ and $x_{n}$. The parameterization of $\tilde{M}$ is gotten by adjoining the equation

$$
p_{\alpha}=-r_{\alpha} / r_{n}=-2\left(\varepsilon_{\alpha} \bar{z}_{\alpha}+F_{\alpha}\right) /\left(i+F^{\prime}\right),
$$

where $F_{\alpha}=\partial F / \partial z_{\alpha}$ and $F^{\prime}=\partial F / \partial x_{n}$. A holomorphic parameterization $T$ of a neighborhood of $(z, p)=(0,0)$ is gotten by allowing $x_{\alpha}, y_{\alpha}$, and $x_{n}$ to assume complex values: $x_{\alpha}=x_{\alpha}^{\prime}+i x_{\alpha}^{\prime \prime}, y_{\alpha}=y_{\alpha}^{\prime}+i y_{\alpha}^{\prime \prime}, x_{n}=x_{n}^{\prime}+i x_{n}^{\prime \prime}$. For convenience let $u=u^{\prime} \dot{+} i u^{\prime \prime}$ denote the coordinate vector. Note that the conjugation $u \rightarrow \bar{u}$ agrees with $\tau$ since they both leave $\tilde{M}$ pointwise fixed.

Consider the image under $T$ of the real ray $x_{\alpha}=y_{\alpha}=x_{n}^{\prime}=0, x_{n}^{\prime \prime}>0$. Since $F$ vanishes to third order at the origin, it follows from the equations for $r$ and $p_{\alpha}$ that this image is a curve transverse to the real hypersurface 
$M \times \mathbf{P}_{n-1}$ and extending into the open set $D \times \mathbf{P}_{n-1}$. We define a $T$ coordinate wedge $W^{+}$as in [3]. Let $e_{0}$ be the real unit vector in the $x_{n}^{\prime \prime}$ direction and $e_{j}, 1 \leqslant j \leqslant 2 n-2$ be real vectors sufficiently close to $e_{0}$ and forming with $e_{0}$ a basis for the $u^{\prime \prime}$ space. Let $V$ be the cone of all $\Sigma t_{i} e_{i}, t_{i}>0$, $\sum t_{i}>0$, intersected with a suitably small ball. Let $E$ be a suitably small ball about the origin in the $u^{\prime}$ space. Put $W^{+}=E+i V$ and $W^{-}=E-i V$. $T\left(W^{+}\right)$will then be contained in $D \times \mathbf{P}_{n-1}$.

Now given the biholomorphic map $f$ as above, let

$$
f_{*}: D \times \mathbf{P}_{n-1} \rightarrow D^{\prime} \times \mathbf{P}_{n-1}
$$

be the map on tangent planes induced by the Jacobian of $f$. Fix $(z, p) \in \tilde{M}$, $\left(z^{\prime}, p^{\prime}\right) \in \tilde{M}^{\prime}$ with $z^{\prime}=f(z)$ and construct coordinate systems $T$ and $T^{\prime}$, respectively about these points. Put $\tilde{f}=T^{\prime-1} \circ f_{*} \circ T$. If $f$ is bicontinuously differentiable then by reflection $\tilde{f}$ can be extended continuously to $W^{+} \cup E$ $\cup W^{-}$. By the continuous version of the edge-of-the-wedge theorem [3], $\tilde{f}$ extends holomorphically to a full neighborhood of the origin. It follows that $f$ extends holomorphically past $z$. This is similar to Lewy's proof [2].

In order to make use of the distributional version of the edge-of-the-wedge theorem, we consider the following condition on $f$.

$$
\begin{aligned}
& f \text { is continuous on } D \cup M \text {. There exists } \\
& \text { a } T \text {-coordinate wedge } W^{+} \cup E \text { with }(z, p) \\
& \in T(E) \text {, such that } \operatorname{Im} \tilde{f}\left(u^{\prime}+i u^{\prime \prime}\right) \\
& \text { approaches zero in the distributional sense } \\
& \text { as } u^{\prime \prime} \text { approaches } 0 \text { and } u \in W^{+} \text {. }
\end{aligned}
$$

The edge-of-the-wedge theorem [3] gives the following:

THEOREM. Let the boundaries $M$ and $M^{\prime}$ be real analytic and nondegenerate. If the biholomorphic map $f$ satisfies the condition (c) at a point $z$ in $M$, then $f$ extends holomorphically to a neighborhood of $z$.

I want to thank C. Fefferman for suggesting that the edge-of-the-wedge theorem might be related to this problem.

\section{REFERENCES}

1. C. Fefferman, The Bergman kernel and biholomorphic mappings of pseudoconvex domains, Invent. Math. 26 (1974), 1-65.

2. H. Lewy, On the boundary behavior of holomorphic mappings, Accad. Naz. dei Lincei, no. 35, 1977.

3. W. Rudin, Lectures on the edge-of-the-wedge theorem, CBMS Regional Conf. Ser. in Math., no. 6, Amer. Math. Soc., Providence, R. I., 1971, pp. 1-30.

4. S. I. Pincuk, On the analytic continuation of holomorphic mappings, Math. Sb. 27 (1975), 375-392.

Department of Mathematics, Princeton University, Princeton, New Jersey 08540 